\title{
Nucleon-Nucleon interaction in a chiral constituent quark model ${ }^{1}$
}

\author{
D. Bartz and Fl. Stancu \\ University of Liège, Institute of Physics, B.5, Sart Tilman, B-4000 Liège 1, Belgium
}

\begin{abstract}
We study the nucleon-nucleon (NN) problem as a six-quark system in a nonrelativistic chiral constituent quark model where the Hamiltonian contains a linear confinement and a pseudoscalar meson (Goldstone boson) exchange interaction between the quarks. This interaction has a long range Yukawa-type part, depending on the mass of the exchanged meson and a short range part, mainly responsible for the good description of the baryon spectra. We calculate the NN potential in the adiabatic approximation as a function of $Z$, the separation distance between the centres of the two three-quark clusters. The orbital part of the six-quark states is constructed either from the usual cluster model states or from molecular orbital single particle states. The latter are more realistic, having proper axially and reflectionally symmetries. In both cases the potential presents an important hard core at short distances, explained through the dominance of the $[51]_{F S}$ configuration. However in the molecular orbital basis the core is less repulsive, as a consequence of the fact that this basis gives a better upper bound for the energy of the six-quark system. We calculate the potential for the ${ }^{3} S_{1}$ and ${ }^{3} S_{0}$ channels with two different parametrizations. We find a small (few $\mathrm{MeV}$ ) attractive pocket for one of these parametrizations. A middle range attraction is simulated by the addition of a $\sigma$-meson exchange interaction between quarks, of a form similar to that of the pseudoscalar meson exchange. The present study is an intermediate, useful step towards dynamical calculations based on the resonating group method.
\end{abstract}

\section{INTRODUCTION}

There have been many attempts to study the nucleon-nucleon interaction starting from a system of six interacting quarks described by a constituent quark model. These models explain the short range repulsion as due to the colour magnetic part of the one gluon exchange (OGE) interaction between quarks and due to quark interchanges between two $3 q$ clusters $[1,2]$. To the OGE interaction it was necessary to add a scalar and a pseudoscalar meson exchange interaction between quarks of different $3 q$ clusters in order to explain the intermediate- and long-range attraction between two nucleons [3-5].

1) Talk presented by D. BARTZ at the International Workshop on Hadron Physics : "Effective Theories of Low Energy QCD", Coimbra, Portugal, September 10-15, 1999. 
In [6] we have calculated the nucleon-nucleon $(N N)$ interaction potential at zeroseparation distance between two three-quark clusters in the frame of a constituent quark model [7-9] where the quarks interact via pseudoscalar meson i.e. Goldstone boson exchange (GBE) instead of OGE. An important motivation in using the GBE model is that it describes well the baryon spectra. In particular, it correctly reproduces the order of positive and negative parity states both for nonstrange [8] and strange [9] baryons where the OGE model has failed.

The underlying symmetry of the GBE model is related to the flavour-spin $S U_{F}(3) \times S U_{S}(2)$ group. Combining it with the $S_{3}$ symmetry, a thorough analysis performed for the $L=1$ baryons [10] has shown that the chiral quark picture leads to more satisfactory fits to the observed baryon spectrum than the OGE models.

The one-pion exchange potential between quarks appears naturally as an iteration of the instanton induced interaction in the t-channel [11]. The meson exchange picture is also supported by explicit QCD latice calculations [12].

Another motivation in using the GBE model is that the exchange interaction contains the basic ingredients required by the $N N$ problem. Its long-range part, required to provide the long-range $N N$ interaction, is a Yukawa-type potential depending on the mass of the exchange meson. Its short-range part, of opposite sign to the long-range one, is mainly responsible for the good description of the baryon spectra [7-9] and also induces a short-range repulsion in the $N N$ system, both in the ${ }^{3} S_{1}$ and ${ }^{1} S_{0}$ channels [13]. This study is an extention of [6] and we calculate here the interaction potential between two $3 q$ clusters as a function of $Z$, the separation distance between the centres of the clusters. This separation distance is a good approximation of the Jacobi relative coordinate between the two clusters. Under this assumption, here we calculate the interaction potential in the adiabatic (Born-Oppenheimer) approximation, as explained below.

A common issue in solving the $N N$ problem is the construction of adequate sixquark basis states. The usual choice is a cluster model basis $[1,2,14]$. In calculating the potential at zero-separation distance, in Ref. [6] we used molecular-type orbitals [15] and compared the results with those based on cluster model single-particle states. The molecular orbitals have the proper axially and reflectionally symmetries and can be constructed from appropriate combinations of two-centre Gaussians. By using molecular orbitals, in Ref. [6] we found that the height of the repulsion reduces by about $22 \%$ and $25 \%$ in the ${ }^{3} S_{1}$ and ${ }^{1} S_{0}$ channels respectively with respect to cluster model results. It is therefore useful to analyse the role of molecular orbitals at distances $Z \neq 0$. By construction, at $Z \rightarrow \infty$ the molecular orbital states are simple parity conserving linear combinations of cluster model states. Their role is expected to be important at short range at least. They also have the advantage of forming an orthogonal and complete basis while the cluster model (two-centre) states are not orthogonal and are overcomplete. For this reason we found that in practice they are more convenient to be used than the cluster model basis, where one must carefully [14] consider the limit $Z \rightarrow 0$. Here too, for the purpose of comparison we perform calculations both in the cluster and the molecular model. 
In the following section we recall the procedure of constructing molecular orbital single-particle states starting from the two-centre Gaussians used in the cluster model calculations. Then the GBE Hamiltonian is presented. The subsequent section is devoted to results obtained for the $N N$ potential. Next we introduce a middle range attraction through a scalar meson exchange interaction between quarks parametrized consistently with the pseudoscalar meson exchange. The last section is devoted to a summary and conclusions.

\section{SINGLE-PARTICLE ORBITALS}

In the cluster model one can define states which in the limit of large intercluster separation $Z$ are right $R$ and left $L$ states

$$
R=\psi\left(\vec{r}-\frac{\vec{Z}}{2}\right) \quad \text { and } \quad L=\psi\left(\vec{r}+\frac{\vec{Z}}{2}\right) .
$$

In the simplest cluster model basis these are ground state harmonic oscillator wave functions centered at $Z / 2$ and $-Z / 2$ respectively. They contain a parameter $\beta$ which is fixed variationally to minimize the nucleon mass described as a $3 q$ cluster within a given Hamiltonian. The states (1) are normalized but are not orthogonal at finite $Z$. They have good parity about their centers but not about their common center $\vec{r}=0$.

From $R$ and $L$ one constructs six-quark states of given orbital symmetry $[f]_{O}$. The totally antisymmetric six-quark states also contain a flavour-spin part of symmetry $[f]_{F S}$ and a colour part of symmetry $[222]_{C}$. In the cluster model the most important basis states [13] for the Hamiltonian described in the following section are

$$
\begin{gathered}
\left|R^{3} L^{3}[6]_{O}[33]_{F S}\right\rangle \\
\left|R^{3} L^{3}[42]_{O}[33]_{F S}\right\rangle \\
\left|R^{3} L^{3}[42]_{O}[51]_{F S}\right\rangle \\
\left|R^{3} L^{3}[42]_{O}[411]_{F S}\right\rangle
\end{gathered}
$$

Harvey [14] has shown that with a proper normalization the symmetry $[6]_{O}$ contains only $s^{6}$ and $[42]_{O}$ only $s^{4} p^{2}$ configurations in the limit $Z \rightarrow 0$.

According to Ref. [15] let us consider also molecular orbital single-particle states. Most generally these are eigenstates of a Hamiltonian $H_{0}$ having axial and reflectional symmetries characteristic to the $N N$ problem. These eigenstates have therefore good parity and good angular momentum projection. As in the cluster model basis where one uses the two lowest states $R$ and $L$, in the molecular orbital basis we also consider the two lowest states, $\sigma$, of positive parity and $\pi$, of negative parity. From these we can construct pseudo-right $r$ and pseudo-left $l$ states as 


$$
\left[\begin{array}{l}
r \\
l
\end{array}\right]=2^{-1 / 2}(\sigma \pm \pi) \quad \text { for all Z, }
$$

where

$$
<r|r>=<l| l>=1,<r \mid l>=0 .
$$

In principle one can obtain molecular orbital single particle states from mean field calculations (see for example [16]). Here we approximate them by good parity, orthonormal states constructed from the cluster model states (1) as

$$
\left[\begin{array}{l}
\sigma \\
\pi
\end{array}\right]=[2(1 \pm<R \mid L>)]^{-1 / 2}(R \pm L)
$$

Such molecular orbitals are a very good approximation to the exact eigenstates of a "two-centre" oscillator frequently used in nuclear physics or occasionally [17] in the calculation of the $N N$ potential. They provide a convenient basis for the first step calculations based on the adiabatic approximation as described below.

Introduced in (6) they give

$$
\left[\begin{array}{c}
r \\
l
\end{array}\right]=\frac{1}{2}\left[\frac{R+L}{(1+<R \mid L>)^{1 / 2}} \pm \frac{R-L}{(1-<R \mid L>)^{1 / 2}}\right] .
$$

At $Z \rightarrow 0$ one has $\sigma \rightarrow s$ and $\pi \rightarrow p$ (with $m=0, \pm 1$ ) where $s$ and $p$ are harmonic oscillator states. Thus in the limit $Z \rightarrow 0$ one has

$$
\left[\begin{array}{l}
r \\
l
\end{array}\right]=2^{1 / 2}(s \pm p)
$$

and at $Z \rightarrow \infty$ one recovers the cluster model basis because $r \rightarrow R$ and $\ell \rightarrow L$.

From $(r, l)$ as well as from $(\sigma, \pi)$ orbitals one can construct six-quark states of required permutation symmetry, as shown in Ref. [15]. In the limit $Z \rightarrow 0$ six-quark states obtained from molecular orbitals contain configurations of type $s^{n} p^{6-n}$ with $n=0,1, \ldots, 6$. For example the $[6]_{O}$ state contains $s^{6}, s^{6} p^{4}, s^{2} p^{4}$ and $p^{6}$ configurations and the $[42]_{O}$ state associated to the $S$-channel contains $s^{4} p^{2}$ and $s^{2} p^{4}$ configurations. This is in contrast to the cluster model basis where $[6]_{O}$ contains only $s^{6}$ and $[42]_{O}$ only $s^{4} p^{2}$ configurations, as mentioned above.

Besides being poorer in $s^{n} p^{6-n}$ configurations, the number of basis states is smaller in the cluster model although we deal with the same $[f]_{O}$ and $[f]_{F S}$ symmetries and the same harmonic oscillator states $s$ and $p$ in both cases. This is due to the existence of three-quark clusters only in the cluster model states, while the molecular basis also allows configurations with five quarks to the left and one to the right, or vice versa, or four quarks to the left and two to the right or vice versa (see Eqs (11)-(19) of [18]). At large separations these states act as "hidden colour" states but at short- and medium-range separation distances they are expected to bring a significant contribution, as we shall see below. The "hidden colour" are states where a $3 q$ cluster in an $s^{3}$ configuration is a colour octet, in contrast to the nucleon which is a colour singlet. Their role is important at short separations but it vanishes at large ones (see e.g. [14]). 


\section{HAMILTONIAN}

The GBE Hamiltonian considered in this study has the form $[8,9]$ :

$$
H=\sum_{i} m_{i}+\sum_{i} \frac{\vec{p}_{i}^{2}}{2 m_{i}}-\frac{\left(\sum_{i} \vec{p}_{i}\right)^{2}}{2 \sum_{i} m_{i}}+\sum_{i<j} V_{\text {conf }}\left(r_{i j}\right)+\sum_{i<j} V_{\chi}\left(r_{i j}\right),
$$

with the linear confining interaction :

$$
V_{\text {conf }}\left(r_{i j}\right)=-\frac{3}{8} \lambda_{i}^{c} \cdot \lambda_{j}^{c}\left(V_{0}+C r_{i j}\right),
$$

and the spin-spin component of the GBE interaction in its $S U_{F}(3)$ form :

$$
\begin{aligned}
V_{\chi}\left(r_{i j}\right) & =\left\{\sum_{F=1}^{3} V_{\pi}\left(r_{i j}\right) \lambda_{i}^{F} \lambda_{j}^{F}\right. \\
& \left.+\sum_{F=4}^{7} V_{K}\left(r_{i j}\right) \lambda_{i}^{F} \lambda_{j}^{F}+V_{\eta}\left(r_{i j}\right) \lambda_{i}^{8} \lambda_{j}^{8}+V_{\eta^{\prime}}\left(r_{i j}\right) \lambda_{i}^{0} \lambda_{j}^{0}\right\} \vec{\sigma}_{i} \cdot \vec{\sigma}_{j},
\end{aligned}
$$

with $\lambda^{0}=\sqrt{2 / 3} \mathbf{1}$, where 1 is the $3 \times 3$ unit matrix. The interaction (13) contains $\gamma=\pi, K, \eta$ and $\eta^{\prime}$ meson-exchange terms and the form of $V_{\gamma}\left(r_{i j}\right)$ is given as the sum of two distinct contributions : a Yukawa-type potential containing the mass of the exchanged meson and a short-range contribution of opposite sign, the role of which is crucial in baryon spectroscopy.

In the parametrization of Ref. [8] the exchange potential due to a meson $\gamma$ has the form

$$
V_{\gamma}^{\mathrm{I}}(r)=\frac{g_{\gamma}^{2}}{4 \pi} \frac{1}{12 m_{i} m_{j}}\left\{\theta\left(r-r_{0}\right) \mu_{\gamma}^{2} \frac{e^{-\mu_{\gamma} r}}{r}-\frac{4}{\sqrt{\pi}} \alpha^{3} \exp \left(-\alpha^{2}\left(r-r_{0}\right)^{2}\right)\right\} .
$$

The shifted Gaussian of Eq. (14) results from a pure phenomenological fit (see below) of the baryon spectrum with

$$
r_{0}=0.43 \mathrm{fm}, \quad \alpha=2.91 \mathrm{fm}^{-1},
$$

For the parametrization of Ref. [9], the potential has the form

$$
V_{\gamma}^{\mathrm{II}}(r)=\frac{g_{\gamma}^{2}}{4 \pi} \frac{1}{12 m_{i} m_{j}}\left\{\mu_{\gamma}^{2} \frac{e^{-\mu_{\gamma} r}}{r}-\lambda_{\gamma}^{2} \frac{e^{-\lambda_{\gamma} r}}{r}\right\} .
$$

where

$$
\Lambda_{\gamma}=\Lambda_{0}+\kappa \mu_{\gamma}, \quad \Lambda_{0}=5.82 \mathrm{fm}^{-1}, \quad \kappa=1.34
$$

In the following, we shall call the form (14) Model I and the form (16) Model II. For a system of $u$ and $d$ quarks only, as it is the case here, the $K$-exchange does not contribute. The apriori determined parameters of the GBE model are the masses 
TABLE 1. Parameters of the Hamiltonian (11-18)

\begin{tabular}{c|c|c|c|c|c}
\hline Model & $V_{0}(\mathrm{MeV})$ & $C\left(\mathrm{fm}^{-2}\right)$ & $g_{8}^{2} / 4 \pi$ & $g_{0}^{2} / 4 \pi$ & Ref. \\
\hline I & 0 & 0.474 & 0.67 & 1.206 & {$[8]$} \\
\hline II & -112 & 0.77 & 1.24 & 2.765 & {$[9]$} \\
\hline
\end{tabular}

$$
m_{u, d}=340 \mathrm{MeV}, \mu_{\pi}=139 \mathrm{MeV}, \mu_{\eta}=547 \mathrm{MeV}, \mu_{\eta^{\prime}}=958 \mathrm{MeV} .
$$

The other parameters are given in Table I.

It is useful to comment on Eqs. (14) and (16). The coupling of pseudoscalar mesons to quarks (or nucleons) gives rise to a two-body interaction potential which contains a Yukawa-type term and a contact term of opposite sign (see e.g. [19]). The second term of (14) or (16) stems from the contact term, regularized with parameters fixed phenomenologically. Certainly more fundamental studies are required to understand this second term and attempts are being made in this direction. The instanton liquid model of the vacuum (for a review see [20]) implies point-like quark-quark interactions. To obtain a realistic description of the hyperfine interaction this interaction has to be iterated in the t-channel [11]. The t-channel iteration admits a meson exchange interpretation [21].

\section{RESULTS}

We diagonalize the Hamiltonian (11)-(18) in the six-quark cluster model basis and in the six-quark molecular orbital basis for values of the separation distance $Z$ up to $2.5 \mathrm{fm}$. Using in each case the lowest eigenvalue, denoted by $\langle H\rangle_{Z}$ we define the $N N$ interaction potential in the adiabatic (Born-Oppenheimer) approximation as

$$
V_{N N}(Z)=\langle H\rangle_{Z}-2 m_{N}-K_{r e l}
$$

Here $m_{N}$ is the nucleon mass obtained as a variational $s^{3}$ solution for a $3 q$ system described by the Hamiltonian (11). The wavefunction has the form $\phi \propto$ $\exp \left[-\left(\rho^{2}+\lambda^{2}\right) / 2 \beta^{2}\right]$ where $\rho=\left(\vec{r}_{1}-\vec{r}_{2}\right) / \sqrt{2}$ and $\vec{\lambda}=\left(\vec{r}_{1}+\vec{r}_{2}-2 \vec{r}_{3}\right) / \sqrt{6}$. The minimum for $m_{N}=\langle H\rangle_{3 q}$ is $970 \mathrm{MeV}$ in the Model I and $1311 \mathrm{MeV}$ in the Model II respectively, reached at the same $\beta$ in both models. The same value of $\beta$ is also used for the $6 \mathrm{q}$ system. This is equivalent with imposing the "stability condition" which is of crucial importance in resonating group method (RGM) calculations $[1,2]$. The quantity $K_{\text {rel }}$ represents the relative kinetic energy of two $3 q$ clusters separated at infinity

$$
K_{r e l}=\frac{3 \hbar^{2}}{4 m \beta^{2}}
$$

where $m$ above and in the following designates the mass of the $u$ or $d$ quark. For our value of $\beta$ this gives $K_{\text {rel }}=0.448 \mathrm{GeV}$. 


\section{Cluster model}

At $Z \rightarrow \infty$ the symmetries corresponding to baryon-baryon channels, namely $[51]_{F S}$ and $[33]_{F S}$, must appear with proper coefficients, as given by Eq. (21). The contribution due to these symmetries must be identical to the contribution of $V_{\chi}$ to two nucleon masses also calculated with the Hamiltonian (11). This is indeed the case. In the total Hamiltonian the contribution of the $[411]_{F S} V_{\chi}$ state tends to infinity when $Z \rightarrow \infty$. Then this state decouples from the rest which is natural because it does not correspond to an asymptotic baryon-baryon channel.

It plays a role at small $Z$ but at large $Z$ its amplitude in the $N N$ wavefunction vanishes, similarly to the "hidden colour" states. Actually, in diagonalizing the total Hamiltonian in the basis (2)-(5) we obtain an $N N$ wavefunction which in the limit $Z \rightarrow \infty$ becomes [14]

$$
\psi_{N N}=\frac{1}{3}\left|[6]_{O}[33]_{F S}\right\rangle+\frac{2}{3}\left|[42]_{O}[33]_{F S}\right\rangle-\frac{2}{3}\left|[42]_{O}[51]_{F S}\right\rangle
$$

The adiabatic potential drawn in Figs. 1 and 2 is defined according to Eq. (19) where $\langle H\rangle_{Z}$ is the lowest eigenvalue resulting from the diagonalization. Fig. 1 corresponds to $S=1, I=0$ in the Model I and Fig. 2 to the same channel in the Model II. Note that from these curves one should subtract $K_{r e l}$ of Eq. (20) in order to obtain the asymptotic value zero for the potential. Similar results are obtained in the $S I=(01)$ channel. One can see that the potential is repulsive at any $Z$ in the Model I, but a small attractive pocket appears in the potential of the Model II (see the zoom in the inside box of Fig. 2).

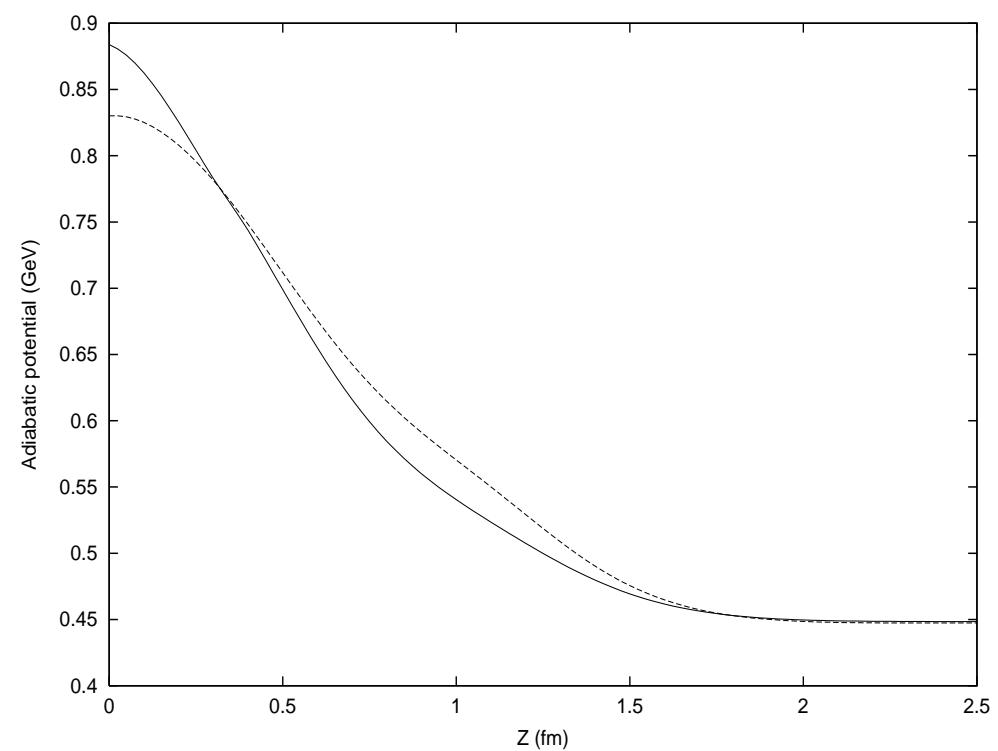

FIGURE 1. Comparison of the adiabatic potential in Model I, for $S I=(10)$, calculated in the cluster model (solid curve) and the molecular orbital basis (dashed curve). 


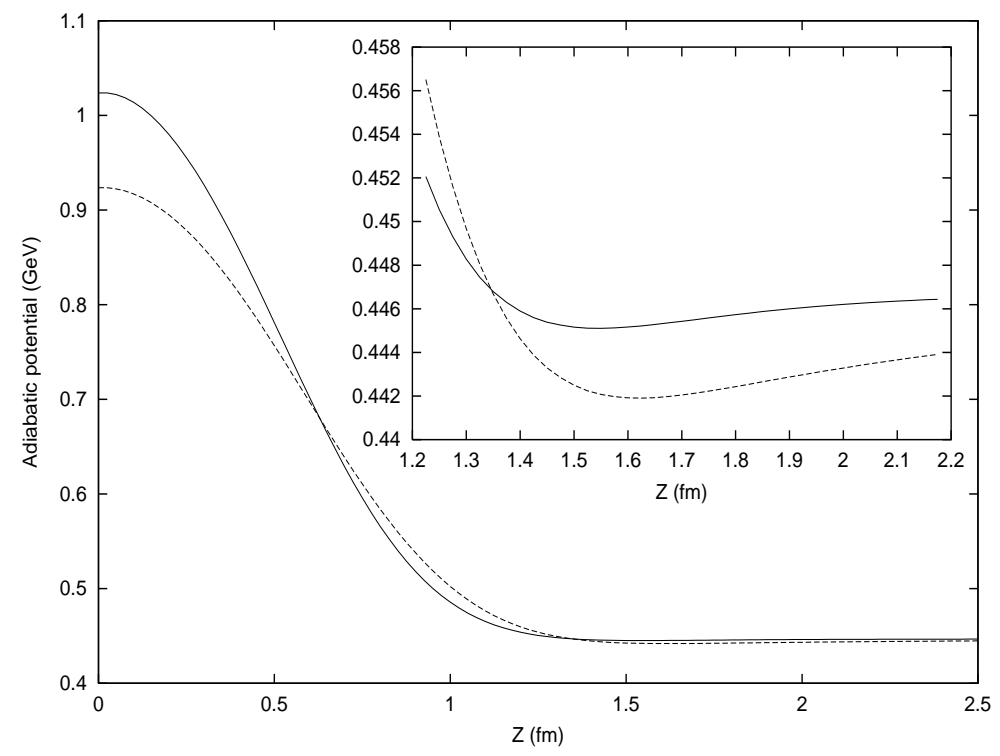

FIGURE 2. Same as Fig. 1 but for the Model II. The upper box represents a zoom of the small attractive pocket.

\section{Molecular orbital basis}

In the molecular orbital basis the asymptotic form of $\psi_{N N}$ is also given by Eq. (21) inasmuch as $r \rightarrow R$ and $l \rightarrow L$ as indicated below Eq. (10).

We diagonalize also the Hamiltonian and use its lowest eigenvalue to obtain the $N N$ potential according to the definition (19). The $S=1, I=0$ case is illustrated for Models I and II in Figs. 1 and 2 respectively, for a comparison with the cluster model basis. The repulsion reduces by about $15 \%$ in the ${ }^{3} S_{1}$ channels when passing from the cluster model basis to the molecular orbital basis. From Figs. 1 and 2 one can see that the molecular orbital basis has an important effect up to about $Z \approx 1.5 \mathrm{fm}$ giving a lower potential at small values of $Z$. For $Z \approx 1 \mathrm{fm}$ it gives a potential larger by few tens of $\mathrm{MeV}$ than the cluster model potential. However there is no attraction at all in the Model I (for details see Ref. [18]).

\section{MIDDLE RANGE ATTRACTION}

In principle we expected some attraction at large $Z$ due to the presence of the Yukawa potential tail in Eq. (14). This is indeed the case in Model II. In Model I, we adopt a consistent procedure assuming that besides the pseudoscalar meson exchange interaction there exists an additional scalar, $\sigma$-meson exchange interaction between quarks. This is in the spirit of the spontaneous chiral symmetry breaking mechanism on which the GBE model is based. The $\sigma$-meson is the chiral partner of the pion and it should be considered explicitly. 
Actually once the one-pion exchange interaction between quarks is admitted, one can inquire about the role of at least two-pion exchanges. Recently it was found [21] that the two-pion exchange also plays a significant role in the quark-quark interaction. It enhances the effect of the isospin dependent spin-spin component of the one-pion exchange interaction and cancels out its tensor component. Apart from that it gives rise to a spin independent central component, which averaged over the isospin wave function of the nucleon it produces an attractive spin independent interaction. These findings also support the introduction of a scalar ( $\sigma$-meson) exchange interaction between quarks as an approximate description of the twopion exchange loops.

For consistency with the parametrization [8] we consider here a scalar quarkquark interaction of the form

$$
V_{\sigma}(r)=\frac{g_{\sigma}^{2}}{4 \pi} \frac{1}{12 m_{i} m_{j}}\left\{\theta\left(r-r_{0}^{\prime}\right) \mu_{\sigma}^{2} \frac{e^{-\mu_{\sigma} r}}{r}-\frac{4}{\sqrt{\pi}} \alpha^{\prime 3} \exp \left(-\alpha^{\prime 2}\left(r-r_{0}^{\prime}\right)^{2}\right)\right\} .
$$

where $\mu_{\sigma}=675 \mathrm{MeV}$ and $r_{0}^{\prime}, \alpha^{\prime}$ and the coupling constant $g_{\sigma}^{2} / 4 \pi$ are arbitrary parameters. In order to be effective at medium-range separation between nucleons we expect this interaction to have $r_{0}^{\prime} \neq r_{0}$ and $\alpha^{\prime} \neq \alpha$. Note that the factor $1 / m_{i} m_{j}$ has only been introduced for dimensional reasons.

We first looked at the baryon spectrum with the same variational parameters as before. The only modification is a shift of the whole spectrum which would correspond to taking $V_{0} \approx-60 \mathrm{MeV}$ in Eq. (12).

For the $6 q$ system we performed calculations in the molecular basis, which is more appropriate than the cluster model basis. We found that the resulting adiabatic

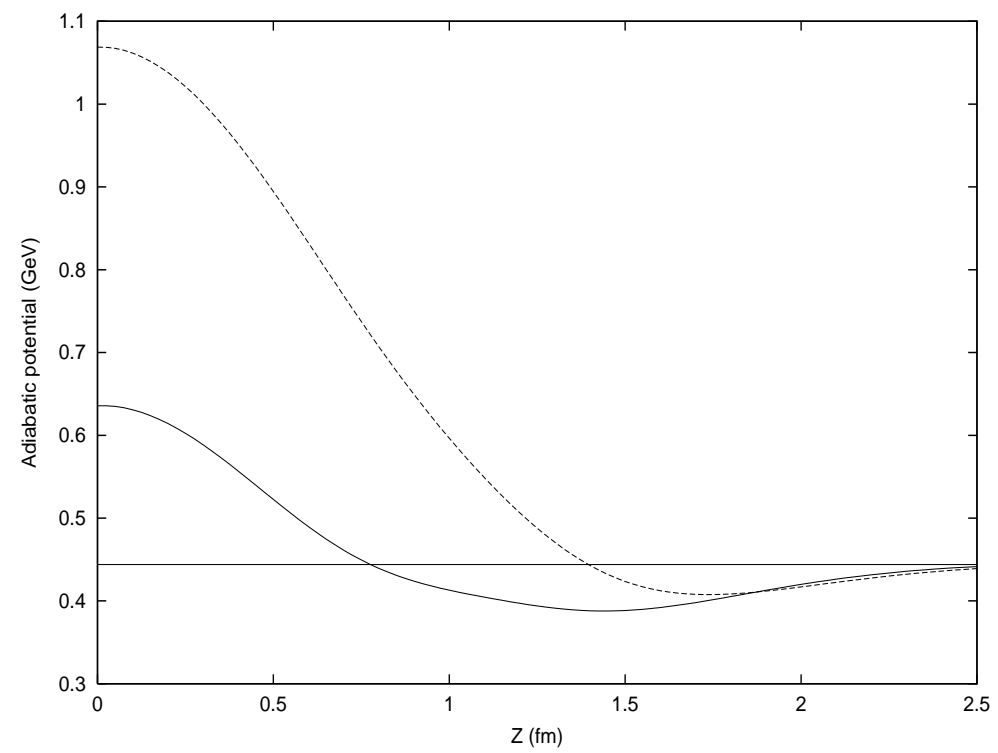

FIGURE 3. The adiabatic potential Model $I$ in the molecular orbital basis for $S I=(10)$ (full curve) and $S I=(01)$ (dashed curve) with pseudoscalar + scalar quark-quark interaction. 
potential is practically insensitive to changes in $\mu_{\sigma}$ and $r_{0}^{\prime}$ but very sensitive to $\alpha^{\prime}$. In Fig. 3 we show results for

$$
r_{0}^{\prime}=0.86 \mathrm{fm}, \quad \alpha^{\prime}=1.47 \mathrm{fm}^{-1}, \quad g_{\sigma}^{2} / 4 \pi=g_{8}^{2} / 4 \pi
$$

One can see that $V_{\sigma}$ produces indeed an attractive pocket, deeper for $S I=(10)$ than for (01), as it should be for the $N N$ problem. The depth of the attraction depends essentially on $\alpha^{\prime}$. The precise values of the parameters entering Eq. (22) should be determined in further RGM calculations. As mentioned above the BornOppenheimer potential is in fact the diagonal RGM kernel. It is interesting that an attractive pocket is seen in this kernel when a $\sigma$-meson exchange interaction is combined with pseudoscalar meson exchange and OGE interactions (hybrid model), the whole being fitted to the $N N$ problem. The same interaction should also be introduced in Model II, but here, our purpose is to show how the $\sigma$-exchange interaction gives some middle-range attraction.

\section{SUMMARY}

We have calculated the $N N$ potential in the adiabatic approximation as a function of $Z$, the separation distance between the centres of the two $3 q$ clusters. We used two different parametrizations of a constituent quark model where quarks interact via pseudoscalar meson exchange. The orbital part of the six-quark states was constructed either from cluster model or molecular orbital single particle states. The latter are more realistic, having the proper axially and reflectionally symmetries. Also technically they are more convenient. We explicitly showed that they are important at small values of $Z$. In particular we found that the $N N$ potential obtained in the molecular orbital basis has a less repulsive core than the one obtained in the cluster model basis. However none of the bases leads to an attractive pocket in one of the parametrizations considered here. We have simulated this attraction by introducing a $\sigma$-meson exchange interaction between quarks.

The present calculations give us an idea about the size and shape of the hard core produced by the GBE interaction. Except for small values of $Z$ the two bases give rather similar potentials. Taking $Z$ as a generator coordinate the following step is to perform a dynamical study based on the resonating group method which will provide phase-shifts to be compared to the experiment. These calculations are underway.

\section{REFERENCES}

1. Oka M. and Yazaki K., Int. Rev. Nucl. Phys., vol. 1 (Quarks and Nuclei, ed. W. Weise), World Scientific, Singapore, p. 490 (1984)

2. Shimizu K., Rep. Prog. Phys. 52 (1989) 1

3. Kusainov A. M., Neudatchin V. G. and Obukhovsky I. T., Phys. Rev. C44 (1991) 1343 
4. Zhang Z., Faessler A., Straub U. and Glozman L. Ya., Nucl. Phys. A578 (1994) 573; Valcarce A., Buchman A., Fernandez F. and Faessler A., Phys. Rev. C50 (1994) 2246

5. Fujiwara Y., Nakamoto C. and Suzuki Y., Phys. Rev. Lett. 76 (1996) 2242; Phys.Rev. C54 (1996) 2180

6. Bartz D. and Stancu Fl., Phys. Rev. C59 (1999) 1756

7. Glozman L. Ya. and Riska D. O., Phys. Rep. 268 (1996) 263

8. Glozman L. Ya., Papp Z. and Plessas W., Phys. Lett. B381 (1996) 311

9. Glozman L. Ya., Papp Z., Plessas W., Varga K. and Wagenbrunn R. F., Nucl. Phys. A623 (1997) 90c

10. Collins H. and Georgi H., Phys. Rev. D59 (1999) 094010

11. Glozman L. Ya. and Varga K., e-print hep-ph/9901439, to appear in Phys. Rev. D

12. Chu M. C. et al., Phys. Rev. D49 (1994) 6039; Negele J. W., Nucl. Phys. Proc. Suppl 73 (1999) 92; Liu K. F. et al., Phys. Rev. D59 (1999) 112001; Aoki S. et al., Phys. Rev. Lett. 82 (1999) 4392

13. Stancu Fl., Pepin S. and Glozman L. Ya., Phys. Rev. C56 (1997) 2779; Phys. Rev. D57 (1998) 4393; Shimizu K. and Glozman L. Ya., e-print nucl-th/9906008

14. Harvey M., Nucl. Phys. A352 (1981) 301; ibid. A481 (1988) 834

15. Stancu Fl. and Wilets L., Phys. Rev. C36 (1987) 726

16. Koepf W., Wilets L., Pepin S. and Stancu Fl., Phys. Rev. C50 (1994) 614

17. Robson D., Phys. Rev. D35 (1987) 1029

18. Bartz D. and Stancu Fl., Phys. Rev. C60 (1999) 055207

19. Brown G. E. and Jackson A. D., The nucleon-nucleon interaction, North Holland, Amsterdam, 1976 p. 5

20. Schäfer T. and Shuryak E. V., Rev. Mod. Phys. 70 (1998) 323

21. Riska D. O. and Brown G. E., Nucl. Phys. A653 (1999) 251

22. Glozman L. Ya., Plessas W., Varga K. and Wagenbrunn R. F., Phys. Rev. D58 (1998) 094030

23. Stancu Fl., Group Theory in Subnuclear Physics, Clarendon Press, Oxford, 1996, chapters 4 and 10 